\title{
THE HIPPOCAMPAL SYSTEM AS THE MANAGER OF NEOCORTICAL DECLARATIVE MEMORY RESOURCES
}

\author{
L. ANDREW COWARD \\ Department of Computer Science, Australian National University, ACT 0200, Australia
}

\begin{abstract}
A model is described in which the hippocampal system receives inputs from cortical columns throughout the neocortex, uses these inputs to determine the most appropriate columns for declarative information recording in response to the current sensory experience, and generates outputs that drive that recording in the selected columns. Evidence in support of the model is described, including physiological connectivity, neuron structures and algorithms, and psychological deficits resulting from damage. Preliminary results from an electronic implementation of the model using leaky integrator neuron models learning via the LTP mechanism are described.
\end{abstract}

\section{Introduction}

Physical damage to the hippocampal system results in a striking combination of memory deficits [1]. This combination includes loss of ability to acquire any new declarative memories (anterograde amnesia), loss of episodic memories for a period of years prior to damage (retrograde amnesia) [2], but preservation of semantic memories including those acquired during the period for which episodic memories are lost [3]. Gen eral intelligence, conversational capabilities and previously acquired skills are retained. New simple skills can be learned, but not skills requiring new declarative memory.

The recommendation architecture model for the mammal brain has been proposed [4] on the basis of theoretical constraints on the architecture of any system which learns to perform a complex combination of behaviours with limited resources. This model has several major subsystems. One subsystem (clustering) is made up of modules that define and detect different groups of similar circumstances within sensory experience. Another subsystem (competition) interprets the detection of any one group of circumstances (indicated by an output from the corresponding module) as a recommendation in favour of a range of different behaviours, each with a specific weight. The competition subsystem determines and implements the behaviour most strongly recommended across all currently detected similarity circumstances. Modules learn their groups gradually in the course of sensory experience, learning taking place in such a way that overlap between similarity circumstances of different 
modules is as small as possible. Module outputs can recommend many different behaviours, hence any changes to a group in the learning process must minimize the undesirable side effects on behaviours already dependent on that group.

Such minimization of side effects means that a group can expand by addition of circumstances similar to those already detected by the group, but with some limited ex ceptions existing circumstances cannot be changed [4]. A corollary of this conclusion is that groups of similarity circumstances cannot in general be evolved to correspond with cognitively unambiguous circumstances like features or cat egories. The pres ence of such cognitive features or cat egories is detected on the basis of predominant recommendation weights across the population of currently detected groups.

Clustering must solve a much more complex problem than problems addressed by statistical clustering theory [5], in which a small set of regularities are sought in a fixed body of data. In clustering, the problem is to find and evolve a set of similarity circumstances useful for managing a very large number of different behaviours within a constantly expanding data set.

On the basis of an extensive range of psychological and physiological evidence, Coward $[4,6]$ has argued that the neocortex can be identified with the clustering subsystem, and the receptive fields of cortical columns correspond with groups of similar circumstances. Subcortical structures including the thalamus and basal ganglia form the competition subsystem that associates groups of similar circumstances and different types of behaviours.

A primary driving force on learning is the requirement that, to ensure a high integrity behaviour, at least a minimum range of recommendations must be generated in response to ev ery sensory input state. This requirem ent means that at least a minimum number of columns must detect their circumstances in every sensory input state. If the minimum is not detected, some columns must expand their receptive fields until the minimum is reached. These expansions are the physiological "trace" of the declarative memory of the input state.

To reduce the risk of undesirable side effects, the expansions in receptive fields must occur in such a way that the total change is as small as possible. A set of columns must therefore be identified which can achieve the minimum total column activity with the least total change. This paper describes a model in which the hippocampal system identifies the appropriate set of neocortical resources in response to each sensory input state.

This cortico-hippocampal model is made up of a hierarchy of descriptions relating phenomena on a neuron level to memory phenomena at a psychological level. At the most detailed level, pyramidal neuron receptive fields are described using leaky integrator neuron models. At a higher level, cortical columns are described using three layers of pyramidal neurons (and some associated interneurons). At a yet higher level, the interactions between cortex and hippocampus are described in terms of column outputs flowing along known anatomical connection pathways. At the highest level, memory deficits are described in terms of damage to specific more detailed capabilities. 


\section{Pyramidal neuron model}

The leaky integrator model for the pyramidal neuron illustrated in figure 1 has been widely used for dynamic modelling of cortical activity [e.g. 7]. The neuron model used for the cortico-hippocampal model is a generalized version of this leaky integrator model which incorporates the concept of staged integration [8].

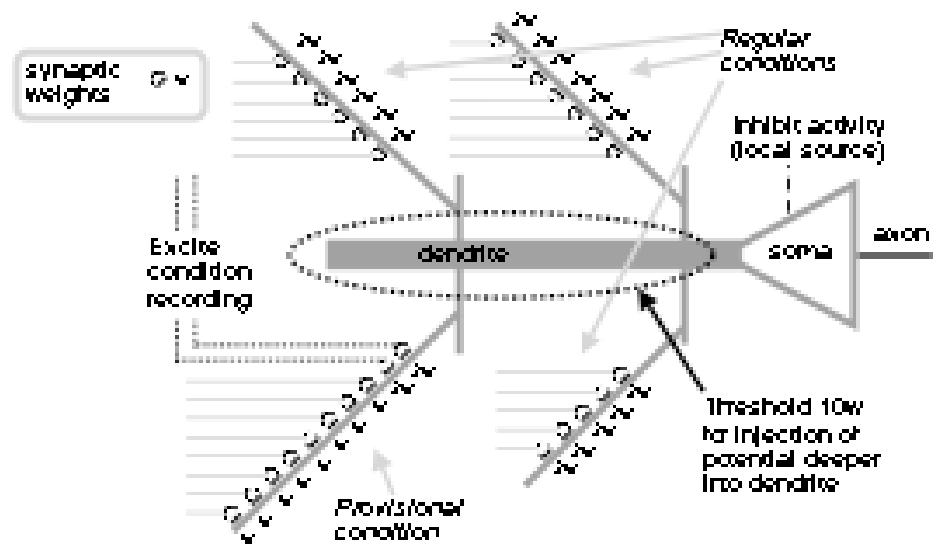

Figure 1. Leaky integrator model for pyramidal neuron with staged integration across its dendritic tree. The LTP mechanism is used to change provisional conditions into regular conditions.

In this staged integration model, the dendritic tree is divided into branches, each branch having a number of synaptic inputs. Action potential spikes directed to a synapse inject potential into the branch proportional to the synaptic strength. This potential rises and decays over a timescale of about 25 milliseconds. If at some point in time the total branch potential derived from all recent synaptic inputs exceeds a threshold, the branch injects a potential into the dendrite. Such injected potentials rise and decay, and only if the total dendritic potential exceeds another threshold will the neuron generate an action potential.

The set of synapses on a branch can be viewed as an information condition within sensory inputs, and the receptive field of the neuron is defined by a group of similar such conditions. The neuron detects its receptive field if a large number of these conditions is present within a relatively short period of time. Such receptive field detections result in an output action potential.

A receptive field expansion is implemented by addition of conditions to the group detected by the neuron. Conditions are added using branches configured with "provisional" conditions, made up of synapses from neurons detecting simpler sensory receptive fields, with total synaptic strengths too small to result in the branch threshold ever being exceeded. Hence the condition 
cannot be detected using the input sensory receptive fields alone. However, the branch has additional inputs labelled "ex cite condition recording" in figure 1. If these inputs are also receiving action potentials, then the branch threshold may be exceeded. If the resultant branch potential injection is followed shortly afterwards by a neuron output, the long term potentiation (LTP) mech anism [9] increas es the synaptic weights of recently active synapses on the branch. This increas e can result in synaptic weights sufficient to lead to future total injections of potential that exceed the branch threshold, independent of the state of the excite condition recording inputs. Hence effectively a new condition has been recorded on the dendrite, slightly expanding the neuron receptive field. This learning algorithm requires ex cite condition recording inputs. Cortical columns generate the information needed to indicate when such inputs are appropriate.

\section{Cortical column model}

The cortical column model illustrated in figure 2 is part of an area of columns that detects receptive fields at one level of complexity in terms of sensory inputs. This area is subject to the requirement discuss ed earlier that it detects at least a minimum number of receptive fields in every sensory input state. A succession of areas detect receptive fields of gradually increasing sensory complexity and pass detections to the next area. An example is the succession of visual areas V1, V2, V4, TEO and TE making up the ventral stream [10].
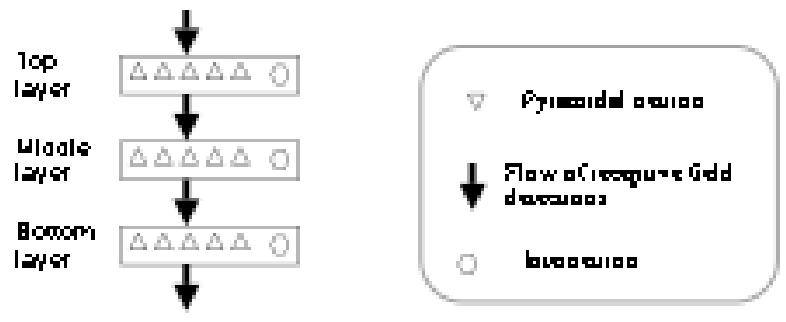

Figure 2. Neocortical column model

There are three layers in the column. The conditions that define the receptive fields of pyramidal neurons in the top layer are combinations of receptive field detections by the columns that provide input to the area in which the column is located. Conditions in middle layer pyramidal neurons are combinations of top layer receptive field detections, and conditions in the bottom layer are combinations of middle layer det ections. Detections by bottom layer neurons are the outputs from the column that are passed to the next area.

The complexity of receptive fields thus gradually increas es from the top to the bottom layer. Pyramidal neurons in the top layer will therefore detect their receptive fields in a relatively wide range of input states, those in the middle 
layer in a somewhat more narrow range, and those in the bottom layer only in the even more narrow range that generate outputs from the column [4].

If the total number of columns generating outputs in the area is less than a required minimum, some columns will need to expand their receptive fields to achieve the minimum. Columns that have no current output (i.e. no activity in their bottom layer) but strong activity in their middle lay er are good candidates for such expansion, because their middle layer activity indicates that the required expansion will be fairly small. A competition is therefore required between the middle layer activity of all the columns in the area to determine the most appropriate columns for such expansion. Pyramidal neurons in the selected columns must then receive inputs that excite condition recording.

One additional factor is relevant to the selection of the most appropriate columns. If all of a specific group of columns have often expanded their receptive fields in the past in response to the same sensory input states, and if many of the group are selected for expansion in response to the current sensory input, the others in the group may well be good candidat es even if their internal activity is a little lower. An optimum competition should therefore take account both of current internal activity and past simultaneous information recording.

Interneurons limit overall activity within columns and within areas. Interneurons in the top layer receive inputs from pyramidals in columns throughout the area, and inhibit pyramidals in their own column layer. Interneu rons in the middle layer receive inputs from their own column and layer, and inhibit the same pyramidals. This inhibition is general to the pyramidal neuron and not specific to individual branches.

\section{Cortico-hippocampal system model}

The major anatomical structures and connectivity paths of the corticohippocampal system in the human brain are illustrated in figure 3. The proposed model interprets these structures and connectivity paths as managing the selection of the most appropriate cortical columns to record information in response to each sensory input situation.

Columns in the perirhinal and parahippocampal cortices have receptive fields corresponding with groups of cortical columns in sensory areas that have expanded their receptive fields at similar times in the past. Entorhinal cortex columns have receptive fields that correspond with larger groups of neocortical columns in many different sensory and polymodal areas that have recorded information in the past at similar times. Information on the current activity of these extensive groups is provided to pyramidal neurons in CA1 and CA3, and to granule cells in the dentate gyrus, by connectivity from the entorhinal cortex.

Dentate gyrus granule cells have receptive fields corresponding with extensive but relatively randomly selected groups of neocortical columns. The receptive fields of pyramidal neu rons in CA3 correspond with extensive groups of neocortical columns that have tended to record information at similar times in 
the past. The receptive fields of CA1 pyramidal neurons correspond with extensive groups of neocortical columns that have recorded information at similar times in the past. There are general similarities between the receptive fields of individual granule cells, CA3 pyramidals and individual CA1 pyramidals in that their receptive fields are all combinations of the same entorhinal cortex outputs. However, in CA1 the fields are sharply focussed in terms of simultaneous past information recording, in CA3 they are less sharply focussed, while the receptive fields of granule cells are relatively unfocussed.

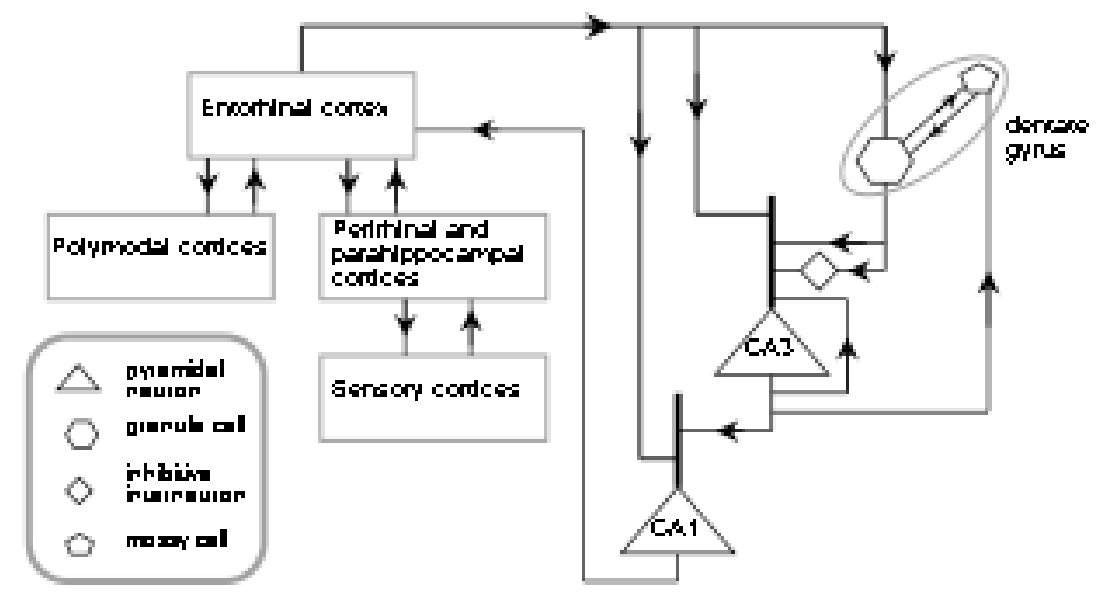

Figure 3. Major connectivity paths of the cortico-hippocampal system. For cortical connectivity see [11]. For entorhinal cortex to hippocampus proper see [12]. For internal hippocampal connectivity see [13].

The receptive fields in CA1, CA3 and the associated cortices need to be defined heuristically, just as sensory receptive fields are defined heuristically. Appropriate signals to drive expansions of receptive fields are therefore required, but the conditions recorded are groups of neocortical columns that expand their receptive fields at the same time. In the model, granule cell outputs drive expansions in CA3 pyramidals, CA3 pyramidal outputs drive expansions in CA1 pyramidals, CA1 pyramidals drive expansions in the entorhinal cort ex, entorhinal cortex outputs drive expansions in the perirhinal, parahippocampal and some polymodal cortices, and the perirhinal and parahippocampal cortex outputs drive expansions in the sensory and other polymodal cortices.

\subsection{Flow of inputs to the hippocampal system}

Activity in the middle layers of a cortical column is an indication of the appropriat eness of the column for receptive field expansion. Outputs from these middle layers in the sensory cortices target perirhinal and parahippocampal 
cortical columns. These perirhinal and parahippocampal columns produce middle layer outputs indicating the degree of internal activity in the groups of sensory columns forming their receptive fields. These middle layer perirhinal and perirhinal outputs target entorhinal cortex columns which produce top and middle layer outputs indicating the degree of internal activity of the groups of groups of sensory columns forming their receptive fields. Pyramidal neurons in CA1 and CA3, and granule cells in the dentate gyrus, receive inputs from the top and middle layers of entorhinal cortex columns.

\subsection{Competition within the dentate gyrus and CA3}

As illustrated in figure 3, within the hippocampus proper there are two positive feedback loops, one in CA3 and the other in the dentate gyrus, with strong links between them. In CA3, pyramidals target large numbers of other pyramidals. In the dentate gyrus, granule cells targ et mossy cells and mossy cells target granule cells. All this internal feedback connectivity is excitatory. Between the two feedback loops, CA3 pyramidals excite dentate gy rus mossy cells, and granule cells both ex cite and (via interneu rons) inhibit CA3 pyramidals. The excitatory connections are the signals exciting CA3 pyramidal receptive field expansions, and the inhibitory connections reduce CA3 pyramidal activity generally. As granule cell activity increas es, inhibition predominates over excitation.

A high degree of activity in the inputs from the entorhinal cortex indicates a strong response to current sensory inputs. Such a strong response indicates that the current input state is fairly familiar and little expansion of receptive fields is required. In response to strong entorhinal cortex input, there will be strong granule cell activity leading to strong CA3 interneuron activity which will cut off CA3 pyramidal activity. Lack of CA3 activity will mean little CA1 activity which will mean little neocortical receptive field expansions.

A smaller degree of entorhinal output means that initially the degree of indirect inhibition of CA3 pyramidals by granule cells will be low. A granule cell also provides outputs that excite condition recording to CA3 pyramidals, these outputs target pyramidals that receive inputs from entorhinal cortex columns similar to those that provide inputs to the granule cell. There will be initial CA3 pyramidal activity from detection of receptive fields as currently defined within entorhinal cortex inputs. Granule cell outputs targetting CA3 pyramidals directly will increase this initial CA3 activity by increasing receptive fields. As CA3 activity increases, the activity of mossy cells (targeted by CA3 pyramidals) increases, and this increases the activity of granule cells. Eventually the increas e in granule cell activity increases the activity of CA3 interneurons to the point at which no further increase in CA3 pyramidals activity occurs.

The activity of CA3 pyramidals will therefore be inversely proportional to the degree of activity in the entorhinal cort ex, which is itself inversely proportional to the novelty of the current sensory input state. The most active CA3 pyramidals will be those with receptive fields corresponding with large 
groups of neocortical columns that currently have strong internal activity. Because new such groups are recorded when the CA3 pyramidals are producing outputs, and such outputs ultimately drive condition recording in those new groups, the CA3 pyramidal receptive fields will evolve to correspond with groups of columns that tended to record information at the same time. The competitive process therefore produces outputs from CA3 pyramidals that favour groups that are both currently active internally and that have tended to record information at the same time in the past.

\subsection{Flow of outputs from the dentate gyrus-CA3 competitive system}

CA3 pyramidals target CA1 pyramidals, both to generally excite those pyramidals and to encourage receptive field expansions. The CA1 pyramidals targetted by a CA3 pyramidal are those receiving similar inputs from the entorhinal cort ex as the CA3 pyramidal. Hence CA1 pyramidals will develop receptive fields that are generally similar to those developed by CA3 pyramidals, but more sharply focussed on groups of columns that record information at the same time.

The outputs of a CA1 pyramidal target pyramidal neurons in columns of the entorhinal cortex from which it derives its inputs. These CA1 outputs both generally excite pyramidals in those columns and drive pyramidal receptive field expansions throughout the columns. The overall effect is that the entorhinal cortex columns most targetted by currently active CA1 pyramidals will produce bottom layer outputs, expanding their receptive fields if necessary. Intern euron activity within those columns limits overall column activity.

Similarly, entorhinal cortex column bottom layer outputs target the perirhinal and parahippocampal columns from which they derive their inputs, and the perirhinal and parahippocampal columns target the sensory cortex columns from which they derive their inputs.

The overall effect is that neocortical columns are selected to expand their receptive fields on the basis of a combination of current internal activity and past simultaneous expansions at the same time as other columns with strong current internal activity. The outputs of the sel ection process both drive current expansions in neocortical sensory receptive fields and evolve pyramidal neuron receptive fields in the hippocampal cortices and hippocampus proper to reflect those current expansions.

\section{Accounting for psychological phenomena}

As described earlier, damage to the hippocampal system results in a striking combination of deficits. The loss of ability to create new declarative memories of any type can be understood as the result of the loss of the outputs from CA1 that drive the receptive field expansions. Observations indicate that damage 
strictly limited to CA1 results in anterograd e amn esia. However, damage to the hippocampal system does not affect the receptive fields of existing neocortical columns, only their ability to change. Those receptive fields and their associated recommendation strengths into subcortical structures will be unaffected. Hence, as observed, hippocampal damage will not affect past skills, general intelligence, conversation skills etc.

There is also an observed loss of episodic memories following hippocampal damage, but not of semantic memories derived from the same time period as the lost episodic memories. [14] has argued that the information mechanisms supporting episodic and semantic memory are qualitatively different.

Episodic memories are constructed by indirect activation of neocortical columns on the basis of past simultaneous inform ation recording. For ex ample, during the experience of a novel event (e.g. watching the Bali bombing on television), there will be receptive field expansions in a wide range of columns. Later, hearing appropriate words (e.g. "Bali bombing") could activate a primary population of columns including a proportion of the columns active during the original event. If this population is evolved by activating a secondary population made up of columns that most often expand ed their receptive fields at the same time as columns in the primary population, and then activating a tertiary population on the same basis and so on, the end point will tend to be an active column population that approximates to the population active during the original experience. Such a final population would have recommendation strengths in favour of speech behaviours describing that original experience etc.

Semantic memories, on the other hand, are based on frequent past simultaneous activity of different groups of columns, generally without receptive field expansions (e.g. groups of visual columns directly activated in response to seeing dogs, and groups of auditory columns directly activated in response to hearing the word "dog").

The hippocampal system role in managing the sel ection of columns that will expand their receptive fields means that the information required to manage episodic memory retrieval in readily available. However, the frequent past simultaneous activity (often without receptive field expansion) information is qualitatively different, and collection of such information by the hippocampal system would corrupt the simultaneous receptive field expansion information required for its primary role. Collection of frequent past simultaneous column activity information must therefore occur in a different neocortical area, and semantic memory will not be affected by hippocampal damage.

\section{Computer implementation of the proposed model}

A version of the cortico-hippocampal model has been implemented by computer, including models for neocortical columns, CA1, CA3 and the dentate 
gyrus. Results for a simple version with just columns and CA1 will be reported here. The flow of time is broken up into one third of a millisecond timeslots, and the states of all the neurons making up the model are recalculated in every timeslot, using new system inputs and the states of all the neurons in the previous timeslot. An action potential occupies one time slot.

One neocortical area with ten columns is modelled. Each column has three layers (figure 2). Each layer has 50 pyramidal neurons and the top two layers each have 10 inhibitory intern eurons. Pyramidal neurons are modelled as two stage leaky integrators. Interneurons are modelled as single stage leaky integrators. Pyramidal neurons in the top layer have 20 branches, each with 25 inputs from randomly selected sensory inputs. Pyramidals in the middle and bottom layers have 10 branches with inputs from 15 randomly selected pyramidal neurons in the preceding layer. Intern eurons targ et pyramidal neurons in the same column and layer. Each top layer interneu ron has randomly selected inputs from pyramidals in the top layer of every column except its own, these interneurons therefore limit the overall activity of the area. Each middle layer interneuron has randomly selected inputs from pyramidals in the middle layer of its own column, these interneurons limit the overall activity of their column.

Learning occurred by an implementation of the LTP algorithm. If a branch injected potential into its dendrite, and its neuron generated an action potential within the next 5 milliseconds, the strengths of recently active synapses to the branch were increased by about $10 \%$. However, unless there were at least five such increases within a 200 millisecond period, the increases were revers ed. In other words, permanent learning only occurred if a provisional condition contributed several times to the production of an action potential by its neuron. Total increases for one synaps e were limited to doubling its original strength.

The hippocampus proper in the simple model has just a CA1 with 10 pyramidal neurons and 10 intern eurons. Each CA1 pyramidal has inputs from a randomly selected set of middle layer pyramidals in a randomly selected set of columns, and targets branches of pyramidals in all layers of the columns from which it receives inputs. The receptive fields of CA1 pyramidals are therefore learned to a very limited degree, and are more comparable with the granul e cell receptive fields in the full model. Overall CA1 activity is limited by interneurons that receive randomly selected inputs from CA1 pyramidals.

Inputs to the cortex emulate the type of inputs that would be received from sensory systems. There are 200 s ensory input streams, and the input state in one time slot can be vi ewed as a 200 element vector indicating the presence or absence of a spike in each input stream. Spikes can occur at different av erage rates in each input stream. Each input stream has a current spike gen eration probability, and the pres ence of a spike is determined randomly on the basis of this probability in each time slot. Hence although there is an average spike rate determined by the probability, spikes occur in randomly selected time slots.

There are different categories of input experiences. These categories could be interpreted, for example, as different categories of visual object. A 
category is defined by a set of spike generation probabilities for the set of 200 input streams. For each category the 200 spike generation probabilities are created by random selection. Category instance presentations last for 200 milliseconds, and are generated for each of the 600 time slots in which the instance is present by using the appropriate category spike generation probabilities. Hence every category instance presentation is different, there is simply a tendency for some input streams to have higher average rates and others to have lower rates for all instances of the same category. A period of 25 milliseconds with no input activity separated each cat egory instance.

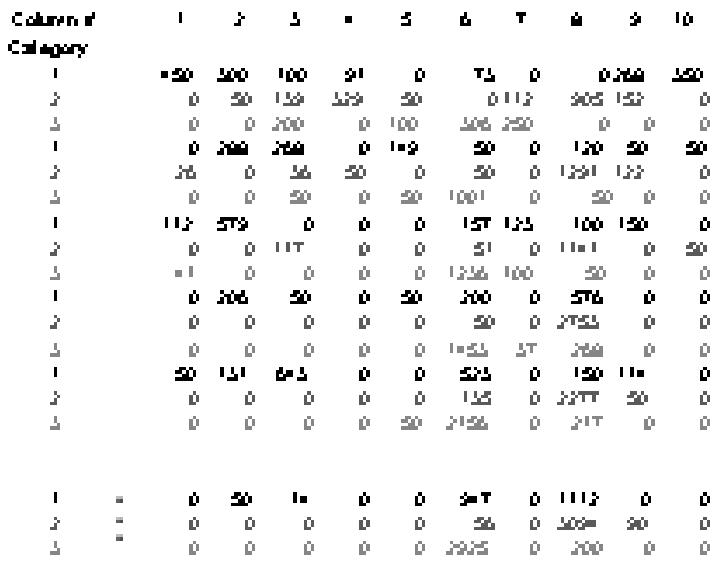

Figure 4. Results of a run of the computer model. The total number of action potentials generated by the pyramidal neurons in the bottom layer of each column during the presentations of 200 millisecond instances of three different categories are shown. The first five and the bst groups of three category instances from a total of 10 (i.e. 30 instance presentations) are shown.

The objective of the computer model is to demonstrate that the model columns can self organize to generate outputs that discriminate between different categories, and that the hippocampal activity improves the effectiveness of the discrimination achieved.

Results of one simulation are shown in figure 4. In this simulation, ten columns were presented with ten sets of different instances of three object categories. The total action potentials generated by the fifty bottom layer pyramidal neurons in response to the first five and the tenth sets of instances are shown. The array of columns rapidly settled into a mode in which category one instances produced activity in columns 6 and 8 , category two instances only in column 6 and category 3 instances only in column 8 . This pattern of response can support an adequate discrimination between the categories from a behavioural point of view [15].

Discrimination effectiveness varied to some degree between runs, but if CA1 signals were removed, this effectiven ess was minimal, with column 
activity tending to be relatively uniform across columns for all categories. The simulation thus demonstrates the feasibility of the model to support column definition. Implementation of the CA3-dentate gyrus system should improve the effectiveness and consistency of the model, and work to test this is under way.

\section{Conclusions}

The proposed model for the cortico-hippocampal system provides a novel but plausible role for the hippocampal system in managing the recording of declarative information in the neocortex. This model makes it possible to understand the operations of the neocortex on multiple levels of detail from physiological to psychological, is consistent with known anatomy and physiology, and provides a straightforward account for the memory deficits that result from physical damage to the hippocampal system. Computer modelling confirms the general feasibility of the model.

\section{References}

1. W. B. Scoville and B. Milner, J. Neur. Neurosurg. Psychiat 20, 11-21 (1957).

2. J. H. Sagar, N. J. Cohen, S. Corkin and J. H. Growden, Ann. New York Acad. Sci. 444, 533-535 (1985).

3. E. A. Kensinger, M. T. Ullman and S. Corkin, Hippocam. 11, 347-360 (2001).

4. L. A. Coward, J. Cogn. Sys. Res. 2(2), 111-156 (2001).

5. Hartigan, J. A. (1985). J. Class. 2, 63 - 76.

6. L. A. Coward, A System Architecture Approach to the Brain: from Neurons to Consciousness. New York: Nova (2005).

7. M. Diesmann, M-O. Gewaltig and A. Aerts en, Nature 402, 529 - 533 (1999).

8. M. Hausser and B. Mel, Curr. Opin. Neurobiol. 13, 372-383 (2003).

9. G-g Bi and M-m Poo, J. Neurosci. 18, 10464-10472 (1998).

10. M. Mishkin, L. G. Ungerleider and K. A. Macko, Trends. Neu rosci. 6, 414 -417 (1983).

11. W. A. Suzuki, Semin. Neurosci. 8, 3-12 (1996).

12. R. Insausti and D. G. Amaral, In G. Paxinos and J. K. Mai editors. The Human Nervous System 871-914 (2004).

13. J. E. Lisman, Neuron 22, 233 - 242 (1999).

14. L. A. Coward, Modelling Language, Cognition and Action, A. Cangelosi, G. Bugmann and R. Botisyuk editors, 311-320 (2005).

15. L. A. Coward, T. D. Gedeon and U. Ratanayake, Lect. Notes Comp. Sci. $3316,458-464$, (2004). 